\title{
CURCUMIN TOPICAL APPLICATION FOR SYMPTOMATIC TREATMENT OF ORAL LICHEN PLANUS: A SYSTEMATIC REVIEW OF EVIDENCE
}

\author{
Gihane Gharib Madkour*, Amal A. Hussine ${ }^{* *}$ and Sandy Shaaban Hassan***
}

\begin{abstract}
Objective: The aim of this systematic review was to assess the clinical effectiveness of topical curcumin, compared to topical corticosteroids for the management of symptomatic oral lichen planus.

Material \& Methods: Three databases were searched including MEDLINE-PubMed, Cochrane-Central \& LILACS as well as Grey literature \& hand-searching till September 2018. Randomized and non-randomized controlled clinical trials relevant to our topic were comprised.

Results: Four studies met our eligibility criteria and were included in this systematic review; 2 randomized and 2 non-randomized controlled trials. All included studies reported clinical improvement of oral lichen planus, in terms of pain alleviation as well as reduction in erythema and ulceration, with the topical use of curcumin. No statistical significant differences were found when comparing the effectiveness of topical curcumin to topical corticosteroids.
\end{abstract}

Conclusion: Topical curcumin application shows promising effects, comparable to topical corticosteroids for the symptomatic treatment of OLP, with no adverse effects.

KEYWORDS: Curcumin, Turmeric, Oral lichen planus, systematic review.

\section{INTRODUCTION}

Medicinal plants have been used in the past decades for the treatment of several human diseases throughout the world ${ }^{1}$. In some developing countries, these medicinal plants are still used as the main medications. As reported by the
World Health Organization (WHO), nearly $80 \%$ of people in developing nations depend mainly on traditional herbal medicines for their primary healthcare ${ }^{2}$. Even in developed countries as United Kingdom, United States, Australia \& Canada, the use of herbal medicines have been growingly established ${ }^{3-6}$. Curcumin is among the most

\footnotetext{
*Associate Professor of Oral Medicine, Diagnosis \& Periodontology, Faculty of Dentistry, Cairo University, Egypt

**Lecturer of Oral Medicine, Diagnosis \& Periodontology, Faculty of Dentistry, Cairo University, Egypt

*** Lecturer of Oral Medicine, Periodontology and Oral Diagnosis, Faculty of Dentistry, Fayoum University, Egypt
} 
powerful well-recognized \& widely studied medicinal herb. Curcumin is an extract from Turmeric which is derived from rhizome of the plant "Curcuma Longa" 7 . Over the last decade, numerous studies have delineated the efficacy \& safety of this magical Curcumin \& have provided a solid basis for investigating its effectiveness in human clinical trials ${ }^{8}$. In fact, Curcumin has been progressively studied for its pleiotropic therapeutic properties such as anti-inflammatory, antioxidant, antimicrobial, antiviral \& anti-carcinogenic properties ${ }^{9}$.

Oral lichen planus (OLP) is a chronic inflammatory \& immunologically mediated disorder, described firstly in 1869 by Wilson ${ }^{10}$. OLP affects most commonly females \& has a prevalence of $2 \%$ among the general population. Clinically, OLP is characterized by usually bilateral mucosal lesions affecting mainly the buccal mucosa, tongue, gingiva $\&$ rarely the palate ${ }^{11}$. OLP may present in different clinical forms including papular, reticular, plaquelike, atrophic or erythematous as well as ulcerative or erosive forms 12, 13. Contemporary treatment modalities of OLP are primarily symptomatic aiming to reduce pain, erythema \& ulceration. Steroids are still the mainstay gold standard for palliative symptomatic treatment of OLP ${ }^{14,15}$. Depending on disease severity, steroids are administered topically, systemically or intra-lesional ${ }^{15}$. Topical steroids application is the preferred one in the management of mild to moderate OLP lesions owing to their fewer side effects ${ }^{16}$. However, prolonged use of topical steroids may lead to secondary candidiasis $\&$ mucosal atrophy ${ }^{17}$. Recently, herbal medicines have been introduced for symptomatic treatment of OLP. Specifically, Curcumin is one of the nutraceuticals that has received a great attention nowadays. Fortunately, Curcumin has been proved to be a safe, non-toxic \& effective alternative for many traditional drugs due to its various therapeutic properties 18, 19. The objective of this systematic review was to elucidate the existing evidence of the efficacy of topical curcumin application for alleviating the symptoms of OLP. Up to our knowledge, this systematic review is the first to explore the efficacy of topical curcumin in OLP.

\section{MATERIALS AND METHODS}

The systematic search \& review process was carried out according to the guidelines of Preferred Reporting Items for Systematic Reviews \& MetaAnalysis (PRISMA) ${ }^{20}$. This review had been registered in the International Prospective Register of Systematic Reviews (PROSPERO), Center for Reviews and Dissemination, University of York on October 25, 2018. The registration number is CRD42018111210.

\section{Focused Question}

Is curcumin topical application effective in alleviating pain \& clinical improvement of symptomatic oral lichen planus patients?

\section{Search strategy}

The authors (G.M. \& A.A.) had searched three electronic databases including MEDLINE-PubMed, Cochrane-Central \& LILACS. In addition, handsearching \& searching in Grey literature was carried out. The search was done in May 2018 \& updated in September 2018. Search terms used in databases were ["Curcumin" OR "Curcuma Longa" OR "Turmeric" OR "Curcuminoids"] AND ["Oral lichen planus" OR "OLP” OR "Erythematous oral lichen planus" OR "Atrophic oral lichen planus" OR "Erosive oral lichen planus" OR "Ulcerative oral lichen planus"].

\section{Eligibility criteria}

Inclusion criteria included: (1) randomized controlled clinical trials (RCTs) \& non-randomized controlled clinical trials (NRCT); (2) articles written in English; (3) population: symptomatic OLP patients; (4) intervention: Curcumin topical application; (5) Control/comparator: corticosteroids topical application; (6) primary outcome: alleviation 
of pain whereas secondary outcome: clinical improvement of OLP in terms of reduction of erythema \& ulceration.

Exclusion criteria were: (1) animal studies; (2) in-vitro studies; (3) narrative reviews; (4) case reports.

\section{Studies Selection}

Initial screening of the title \& abstract of studies was performed by the two reviewers G.M. \& A.A. separately. Full-text screening was then carried out by the same reviewers $\&$ any disagreement between them was solved by discussion. Studies fulfilling the eligibility criteria were included.

\section{Quality Assessment}

Assessment of the methodological quality of selected studies was performed by the authors G.M. \& S.H. independently. Assessment of risk of bias was accomplished utilizing the Cochrane Risk of Bias tool as clarified in the Cochrane handbook for RCTs ${ }^{21}$. For assessment of risk of bias for non-randomized studies of interventions, Cochrane ROBINS-I tool was used ${ }^{22}$. Domains assessed for every included study encompassed sequence generation; allocation concealment; blinding of participants, personnel \& outcome assessors; incomplete outcome data; selective outcome reporting and other source of bias. When all these domains are fulfilled, the study is categorized as being a study with low risk of bias. If one domain is missing, the study will have a moderate risk of bias. Two or more missing domains categorize the study as having a high risk of bias ${ }^{21}$.

\section{Data Extraction}

Selected articles were processed for data extraction. Data extracted from selected papers included: the study design \& duration of the study; sample size; demographic characteristics of included patients; OLP forms; curcumin formulations \& applications; the control or comparator used; clinical parameters assessed \& the authors' conclusions.
Extracted data were then tabulated. (Table 1)

In fact, data extracted from included studies showed substantial heterogeneity in several aspects, thus, we couldn't perform a meta-analysis. Hence, extracted data from selected studies were abridged $\&$ exhibited in an illustrative method.

\section{RESULTS}

After our initial search \& removal of duplicates, 18 papers were discovered. Screening the titles $\&$ abstracts resulted in exclusion of 4 papers \& 14 papers were then selected. Following full-text reading, 10 papers were excluded as one paper ${ }^{17}$ was a Narrative Review, two papers ${ }^{23,24}$ were Pilot studies with only one OLP group \& no control or comparator group, one paper ${ }^{25}$ was a case report, one retrospective cohort study ${ }^{26}$, four papers ${ }^{27-30}$ used systemic administration of Curcumin \& one paper ${ }^{31}$ used a different comparator that did not match our eligibility criteria. Only 4 articles met the eligibility criteria ${ }^{32-35}$. The number of papers recognized at different stages of reviewing is shown in the PRISMA flowchart (Figure 1).

Among the 4 included studies, 2 studies were randomized controlled trials; Keshari et al., ${ }^{32}$ and Kia et al., ${ }^{33}$ and the other 2 studies were nonrandomized controlled trials; Thomas et al., ${ }^{34}$ and Nosratzehi et al., ${ }^{35}$. In the 4 included studies, total number of participating subjects was 192 (130 females \& 62 males) with age ranges from 20-78 years. All participating subjects were suffering from symptomatic OLP. In their studies, Keshari et al., ${ }^{32}$ and Kia et al., ${ }^{33}$ as well as Nosratzehi et al., ${ }^{35}$ enrolled patients with atrophic and/or erosive OLP types. Thomas et al., ${ }^{34}$ enrolled only patients with erosive OLP.

In all included studies ${ }^{32-35}$ topical application of curcumin was compared to topical corticosteroids application for symptomatic treatment of OLP. However, different formulations \& concentrations of both curcumin as well as corticosteroids were 


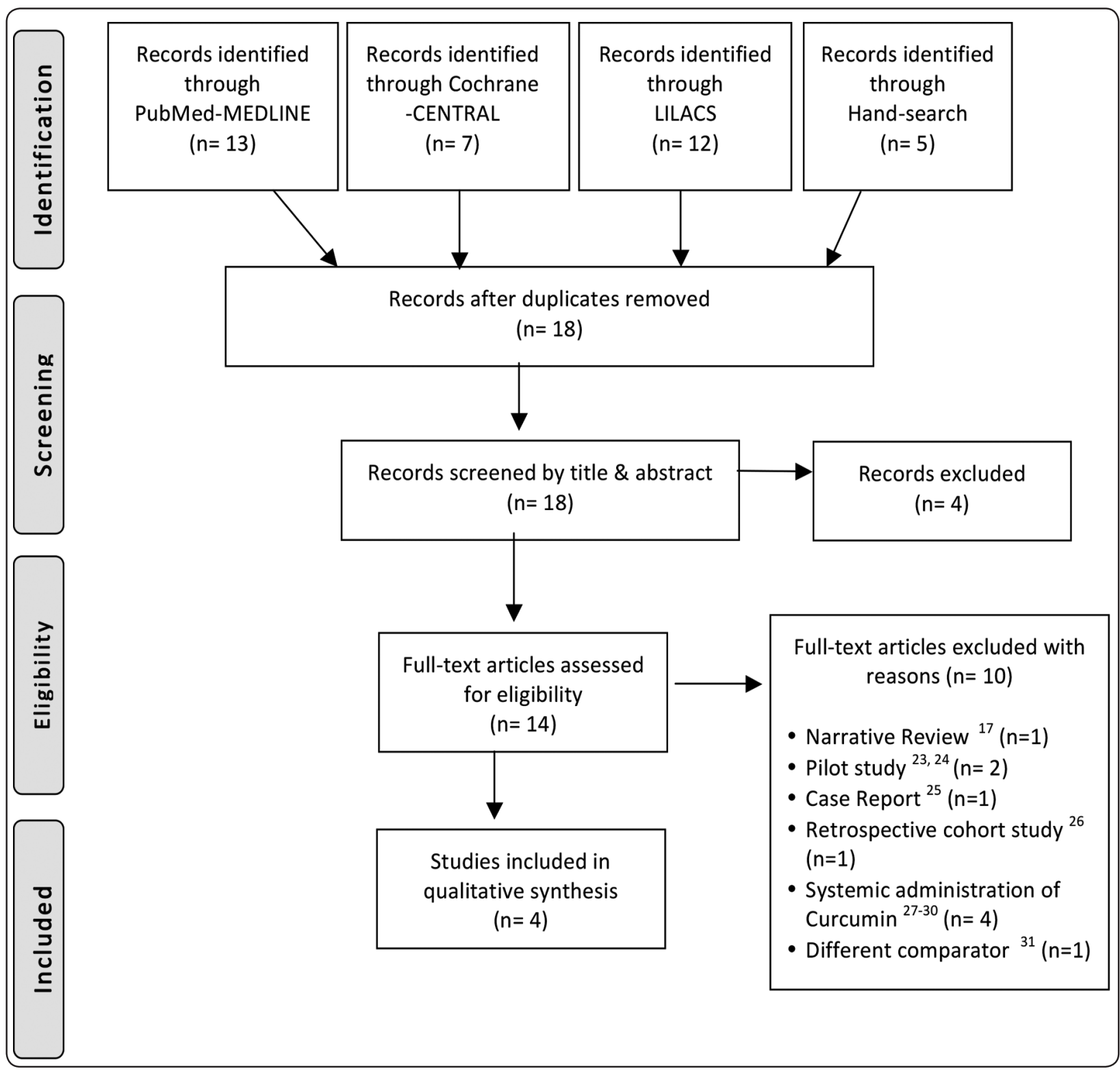

Fig. (1) Preferred Reporting Items for Systematic Reviews \& Meta-analysis (PRISMA) flow Diagram showing number of studies

applied. Specifically, Keshari et al., ${ }^{32}$ and Thomas et al., ${ }^{34}$ used commercially available curcumin $1 \%$ oral gel (Curenext oral gel) whereas Kia et al., ${ }^{33}$ used curcumin $5 \%$ oral paste. Nosratzehi et al., ${ }^{35}$ used curcumin mucoadhesive paste prepared in the faculty of Pharmaceutics, Tabriz University of Medical Sciences in Iran and the authors didn't report the exact concentration of curcumin. Curcumin was applied three times daily after meals in all included studies. Topical corticosteroids application was used as comparator in the form of triamcinolone acetonide oral paste $0.1 \%$ in Keshari et al., ${ }^{32} \mathrm{Kia}$ et al., ${ }^{33}$ and Thomas et al., ${ }^{34}$ studies unlike Nosratzehi et al. ${ }^{35}$ who used Betamethasone $0.1 \%$ local steroid lotion. Topical corticosteroids were applied three times daily in all included studies. The duration of the intervention varied between included studies; two weeks in Keshari et al. ${ }^{32}$ study, four weeks in 
Kia et al., ${ }^{33}$ study and three months in both Thomas et al., ${ }^{34}$ and Nosratzehi et al., ${ }^{35}$ studies.

Regarding clinical parameters assessed, Keshari et al., ${ }^{32}$ used pain score ${ }^{36}$ in the form of numerical rating scale ranging from 0 to 10 and erythema score ${ }^{36}$ ranging from 0 to 3 where $0=$ Normal; $1=$ Mild erythema; 2 = Moderate erythema; $3=$ Severe erythema, as well as ulceration score ${ }^{36}$ where $0=$ No ulcerations; 1 = between 0 and $0.25 \mathrm{~cm}^{2} ; 2=$ between 0.25 and $1 \mathrm{~cm}^{2} ; 3=\geq 1 \mathrm{~cm}^{2}$. On the other hand, Kia et al., ${ }^{33}$ assessed the severity of pain using visual analog scale ${ }^{37}$ ranging from 0 to 10 as well as size of the lesions using Thongprasom score ${ }^{38}$ where $0=$ No lesion; $1=$ Mild white striae, no erythematous area; $2=$ White striae with atrophic area less than $1 \mathrm{~cm}^{2} ; 3=$ White striae with atrophic area more than $1 \mathrm{~cm}^{2} ; 4=$ White striae with ulcerative area less than $1 \mathrm{~cm}^{2} ; 5=$ White striae with ulcerative area more than $1 \mathrm{~cm}^{2}$. Thomas et al., ${ }^{34}$ assessed burning sensation by means of numerical rating scale from 0 to 10 ${ }^{39}$ and they evaluated clinical signs using Modified Oral Mucositis Index developed by Schubert et al., ${ }^{40}$. Nosratzehi et al., ${ }^{35}$ evaluated the size of lesions using Thongprasom score ${ }^{38}$, severity of pain and burning sensation using a $10 \mathrm{~cm}$ visual analog scale ${ }^{41}$ and they also assessed severity index \& pain index ${ }^{42}$.

Concerning pain reduction which is the primary outcome assessed in all included studies, Keshari et al., ${ }^{32}$ reported statistically significant reduction in pain scores from baseline to follow-up in both curcumin study group and triamcinolone control group $(\mathrm{P}=0.005$ and $\mathrm{P}=0.016$ respectively $)$. However, when comparing the difference in decrease of pain scores between the two studied groups, insignificant statistical difference was observed $(\mathrm{P}=0.697)$. Similarly, Kia et al., ${ }^{33}$ reported significant pain reduction in both curcumin $\&$ triamcinolone groups. The authors revealed that complete remission was observed in $36 \%$ \& $32 \%$ of the cases respectively; good response in $16 \%$ \& $32 \%$ respectively; poor response in $24 \%$ \& $16 \%$ respectively and no response to treatment in $24 \%$ \& $20 \%$ respectively. No statistical significant difference was detected between the two studied groups ( $\mathrm{P}=0.17$ at baseline; $\mathrm{P}=0.3$ at 2 weeks; $\mathrm{P}=0.46$ at 4 weeks follow up). Similar results were reported by Nosratzehi et al., ${ }^{35}$ who showed statistically significant decrease in pain severity in both studied groups from baseline to 12 weeks follow up $(p<0.05)$. However, no statistical difference was found between the curcumin \& corticosteroid groups $(\mathrm{P}>0.05)$. Thomas et al., ${ }^{34}$ showed in their study results significant reduction in burning sensation among their three studied groups from baseline to three months follow up; triamcinolone (77.39\%); curcumin applied 3 times daily (54.4\%) and curcumin applied 6 times daily $(64.99 \%)$. Triamcinolone group showed the highest reduction in burning sensation followed by curcumin application 6 times daily. The authors reported that reduction in burning sensation among the triamcinolone group \& curcumin application 6 times daily group was almost comparable.

Clinical improvement of OLP in terms of reduction of erythema \& ulceration was reported in all included studies. In fact, Keshari et al., 32 demonstrated statistically significant reduction in erythema in both curcumin and triamcinolone acetonide groups $(\mathrm{P}=0.000$ and $\mathrm{P}=0.007$ respectively). Moreover, the authors reported a statistically significant improvement in erythema among the curcumin group when comparing the mean erythema scores of curcumin and triamcinolone acetonide groups $(\mathrm{P}=0.002)$. In addition, a statistically significant reduction in the ulceration scores was shown among the curcumin group from baseline to follow up $(\mathrm{P}=0.041)$, while the ulceration scores in the triamcinolone acetonide group did not show significant reduction $(\mathrm{P}=0.674)$. Though, comparing the mean ulceration scores of both studied groups revealed statistically insignificant results $(\mathrm{P}=0.291)$. 


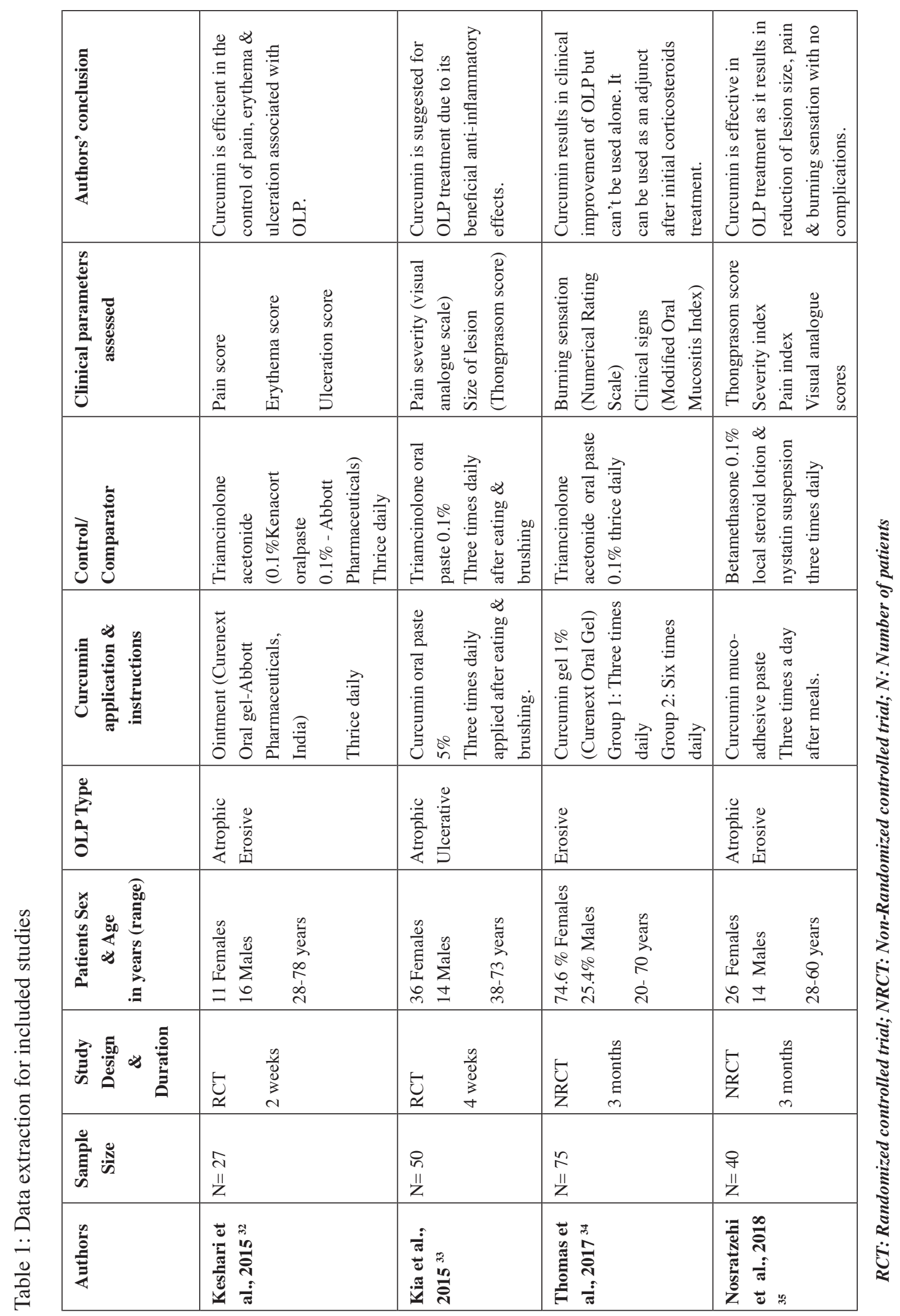


Kia et al., ${ }^{33}$ reported decreased mean Thongprasom score in curcumin group from $3.88 \pm 0.78$ at baseline, to $2.64 \pm 1.29$ at final follow up. In triamcinolone group, mean Thongprasom score decreased from $3.95 \pm 1.07$ at baseline, to $2.95 \pm 0.97$ at final follow up. However, the authors reported no significant difference between the two groups ${ }^{33}$.

Thomas et al., ${ }^{34}$ showed in their results a significant decrease in erythema \& ulceration among curcumin \& triamcinolone acetonide studied groups using the Modified Oral Mucositis Index ( $<<0.001)$. They clarified that this reduction was the highest among triamcinolone group (67.8\%) followed by curcumin applied six times daily group (58.36\%). The lowest reduction was observed in curcumin applied three times daily group (46.6\%).

Nosratzehi et al., ${ }^{35}$ observed significant decrease in OLP lesion sizes within both curcumin \& corticosteroid groups along all follow up visits $(2.45 \pm 1.1$ to $0.75 \pm 0.34$ and $2.61 \pm 1.2$ to $0.91 \pm 0.46$ respectively) $(\mathrm{p}<0.05)$. Yet, intergroup comparison revealed no significant difference observed between curcumin and corticosteroid groups $(\mathrm{P}>0.05)$. In addition, based on Thongprasom classification, the authors reported improvement in the two studied groups along follow-up visits. No statistical difference was reported between the two groups. The authors stated that curcumin had a comparable effect to corticosteroids ${ }^{35}$.

Assessment of risk of bias of all included studies revealed that only Kia et al, ${ }^{33}$ study had moderate risk of bias while Keshari et al., ${ }^{32}$ and Thomas et al., ${ }^{34}$ as well as Nosratzehi et al., ${ }^{35}$ studies had high risk of bias.

\section{DISCUSSION}

Although topical corticosteroids remain the backbone for symptomatic treatment of OLP, alternative treatment modalities are greatly considered nowadays, owing to the deficiency of robust evidence regarding existing therapeutic modalities ${ }^{43-45}$. In fact, Thongprasom et al., $(2011)^{43}$, Cheng el al., (2012) ${ }^{44}$ and Lodi et al., $(2012)^{45}$ conducted three systematic reviews to assess the effectiveness of existing treatment modalities for symptomatic OLP. Unfortunately, they all reported insufficient evidence supporting the efficacy of existing treatment modalities when compared to placebo or even when comparing two active interventions, including steroids. Indeed, management of OLP represents a great challenge as, till now, there is no treatment that leads to complete cure of the disease. Only symptomatic treatments alleviating pain, reducing erythema and ulcerations are available ${ }^{14}$. New therapeutic modalities including topical pimecrolimus ${ }^{46}$, tacrolimus ${ }^{47}$, thalidomide 48, amlexanox ${ }^{49}$, aloe vera ${ }^{50}$ and curcumin ${ }^{24,32-35}$ have been suggested. Curcumin is among the new therapeutic modalities for managing OLP with great promising results ${ }^{14,17}$. The universal approval and usage of herbal medicines keep on assuming an exponential rise nowadays. Therefore, the aim of this systematic review was to assess the standing evidence regarding the effectiveness of topical curcumin as a new alternative for symptomatic treatment of OLP.

This systematic review comprised four clinical trials; 2 randomized controlled trials (Keshari et al., $32 \&$ Kia et al., 33) and 2 non-randomized controlled trials (Thomas et al., 34 \& Nosratzehi et al., 35). The four trials investigated the efficacy of topical application of curcumin, compared to topical corticosteroids, for the symptomatic treatment of patients with OLP. The total number of included patients is 192 and the duration of intervention ranged from 2 weeks to 3 months. The included studies showed considerable heterogeneity regarding study design, curcumin formulation and dosage, type and dose of corticosteroid comparator as well as the duration of treatment, thus we couldn't perform meta-analysis. Interestingly, results of all included studies 32-35 revealed improvement 
of OLP symptoms, in terms of pain alleviation as well as reduction in erythema and ulceration, with the use of topical curcumin. Precisely, Keshari et al., 32 concluded that curcumin can be used as an alternative to corticosteroids in OLP management. However, the authors recommended further trials to evaluate, pre- and post-treatment, histological parameters to determine the effectiveness of topical curcumin at the cellular level. Likewise, Kia et al., 33 and Nosratzehi et al., 35 suggested topical curcumin application for OLP management owing to its fortunate anti-inflammatory properties.

Although Thomas et al., ${ }^{34}$ reported clinical improvement of OLP with topical curcumin oral gel; they concluded that curcumin cannot be utilized as a substitute for corticosteroids. They proposed the use of curcumin after an initial regimen of topical steroids. It is imperative here to highlight the potential association between the different doses/formulations of curcumin used and its therapeutic effects in the included clinical trials. For instance, Thomas et al., ${ }^{34}$ used $1 \%$ curcumin oral gel whereas Kia et al., ${ }^{33}$ used 5\% curcumin oral paste. This may explain the different conclusions reported by the two studies. In addition, it may be suggested that higher concentration of curcumin produces superior favorable therapeutic results. Fortunately, all included studies did not report any adverse effects associated with the application of curcumin topically. In fact, this represents the major advantage of curcumin compared to corticosteroids. Corticosteroids have considerable adverse effects, even when applied topically. Actually, prolonged use of topical corticosteroids may lead to mucosal atrophy and oral candidal infection 16,17. Given the chronic nature of OLP and the need for long treatment periods, curcumin seems to be more advantageous and safer compared to steroids ${ }^{33}$.

To our knowledge, this is the first systematic review evaluating the available clinical evidence concerning the therapeutic use of topical curcumin for the management of symptomatic OLP. Assessment of risk of bias revealed that among the included four studies, only kia et al, ${ }^{33}$ had moderate risk of bias. Keshari et al. ${ }^{32}$, Thomas et al. ${ }^{34}$ and Nosratzehi et al., ${ }^{35}$ studies had high risk of bias. Thus, although we found clinical evidence in the literature that topical curcumin is as effective as topical corticosteroids in managing symptomatic OLP, yet the existing body of evidence is weak.

The main limitation that encountered us in this systematic review was the little number of conducted randomized controlled trials. Other limitations comprised small sample sizes, different formulations and concentrations of curcumin as well as the outcome assessment measures. Moreover, we only included studies in English. Hence, we may have overlooked other studies published in different languages.

\section{CONCLUSION}

Based upon the existing evidence, the authors concluded that topical curcumin application shows promising effects comparable to topical corticosteroids for the symptomatic treatment of OLP with no adverse effects. We advocate more randomized controlled trials to be conducted with large sample sizes and standardized curcumin topical preparations and dosage, to increase the body of evidence concerning the effectiveness of curcumin in OLP.

\section{CONFLICT OF INTEREST}

The authors declare no conflict of interest.

\section{REFERENCES}

1. Karunamoorthi K, Jegajeevanram K, Vijayalakshmi J and Mengistie E (2013): Traditional Medicinal Plants: A Source of Phytotherapeutic Modality in ResourceConstrained Health Care Settings. Journal of EvidenceBased Complementary \& Alternative Medicine; 18(1): 6774. DOI: $10.1177 / 2156587212460241$ 
2. Ekor M (2014): The growing use of herbal medicines: issues relating to adverse reactions and challenges in monitoring safety. Front Pharmacol; 4:177. doi: 10.3389/ fphar.2013.00177

3. Anquez-Traxler C (2011): The legal and regulatory framework of herbal medicinal products in the European Union: a focus on the traditional herbal medicines category. Therapeutic Innovation \& Regulatory Science; 45(1): 1523. DOi: $10.1177 / 009286151104500102$

4. Eisenberg DM, Davis RB, Ettner SL, Appel S, Wilkey S, Van RM (1998): Trends in alternative medicine use in the United States, 1990-1997: results of a follow-up national survey. JAMA; 280(18): 1569-1575. doi: 10.1001/ jama.280.18.1569.

5. Xue CC, Zhang AL, Lin V, Da CC, Story DF (2007): Complementary and alternative medicine use in Australia: a national population-based survey. J Altern Complement Med; 13(6): 643-650. doi: 10.1089/acm.2006.6355.

6. McFarland B, Bigelow D, Zani B, Newsom J, Kaplan M (2002): Complementary and alternative medicine use in Canada and the United States. Am J Public Health; 92(10): 1616-1618. doi: 10.2105/AJPH.92.10.1616.

7. Gupta SC, Patchva S, Koh W, Aggarwal BB (2012): Discovery of curcumin, a component of golden spice, and its miraculous biological activities. Clin Exp Pharmacol Physiol.; 39(3): 283-99. doi:10.1111/j.14401681.2011.05648.x.

8. Gupta SC, Patchva S, Aggarwal BB (2013): Therapeutic roles of curcumin: lessons learned from clinical trials. AAPS J; 15(1): 195-218. doi: 10.1208/s12248-012-9432-8.

9. Cikrikci S, Mozioglu E, Yilmaz H (2008): Biological activity of curcuminoids isolated from Curcuma longa. Rec Nat Prod;2(1):19-24.

10. Wilson E (1869): On lichen planus. J Cutan Med Dis Skin; 3:117-132.

11. Zakrzewska JM (2001) Re: Mollaoglu N. Oral lichen planus: a review. Br J Oral Maxillofac Surg 2000; 38: 370377. Br J Oral Maxillofac Surg 39: 407.

12. McCartan BE \& Healy CM (2008): The reported prevalence of oral lichen planus: A review and critique. Journal of Oral Pathology and Medicine; 37: 447-453.

13. Scully C \& Carrozzo M (2008): Oral mucosal disease: Lichen planus. British Journal of Oral and Maxillofacial Surgery; 46: 15-21.
14. Thongprasom K, Prapinjumrune C, Carrozzo M (2013): Novel therapies for oral lichen planus. J Oral Pathol Med. Nov;42(10):721-7. doi: 10.1111/jop.12083.

15. Patil S, Khandelwal S, Sinha N, Kaswan S, Rahman F and Tipu S (2016): Treatment modalities of oral lichen planus: An update. J Oral Diag; 01: 47-52.

16. Farhi D, Dupin N (2010): Pathophysiology, etiologic factors, and clinical management of oral lichen planus, part I: facts and controversies. Clin Dermatol 28: 100-108.

17. Sneha S, Ul Nisa S and Mhapuskar A (2017): Curcumin - a novel ayurvedic treatment for oral lichen planus. International Journal of Current Medical and Pharmaceutical Research; 3(03): 1507-1511.

18. Cheng AL, Hsu CH, Lin JK, Hsu MM, Ho YF, Shen TS et al. (2001): Phase I clinical trial of curcumin, a chemopreventive agent, in patients with high risk or pre-malignant lesions. Anticancer Res; 21(4b): 2895-900.

19. Farid RM (2016): A Focus on Curcumin Local Application in Oral Diseases Management: Mini Review. IOSR Journal of Pharmacy; 6(1): 30-40.

20. Moher D, Liberati A, Tetzlaff J, Altman D, The PRISMA Group (2009): Preferred Reporting Items for Systematic Reviews and Meta-Analyses: The PRISMA Statement. Ann Intern Med.2009; 151:264-269. doi:10.7326/00034819- 151-4-200908180-00135

21. Higgins JP and Green S (2008): Cochrane handbook for systematic reviews of interventions. Version 5.1.0, Available at: http://handbook-5-1.cochrane.org/.

22. Sterne JAC, Hernán MA, Reeves BC, Savović J, Berkman ND et al. (2016): ROBINS-I: a tool for assessing risk of bias in non-randomized studies of interventions. BMJ; 355; i4919; doi: 10.1136/bmj.i4919.

23. Kia SJ, Basirat M, Estakhr L (2017): The Effect of Oral Curcumin on Pain and Clinical Appearance of Oral Lichen Planus. 3DJ; 6(1):1-7.

24. Singh V, Pal M, Gupta S, Tiwari SK, Malkunje L, Das S (2013): Turmeric - A new treatment option for lichen planus: A pilot study. Natl J Maxillofac Surg; 4(2):198201. doi: 10.4103/0975-5950.127651. PubMed PMID: 24665176; PubMed Central PMCID: PMC3961895.

25. Prasad S, Solanki S, Chinmaya BR, Tandon S, Ashwini B (2014): The Magic of Herbal Curcumin Therapy in Recurrent Oral Lichen Planus. American Journal of Ethnomedicine; 1(1): 096-101. 
26. Chainani-Wu N, Collins K, Silverman S Jr (2012): Use of curcuminoids in a cohort of patients with oral lichen planus, an autoimmune disease. Phytomedicine; 19(5):418-23. doi: 10.1016/j.phymed.2011.11.005. Epub 2012 Feb 2. PubMed PMID: 22305276.

27. Chainani-Wu N, Silverman S Jr, Reingold A, Bostrom A, Mc Culloch C, Lozada-Nur F, Weintraub J. A randomized, placebo-controlled, double-blind clinical trial of curcuminoids in oral lichen planus. Phytomedicine. 2007 Aug;14(7-8):437-46. Epub 2007 Jul 2. PubMed PMID: 17604143 .

28. Chainani-Wu N, Madden E, Lozada-Nur F, Silverman S Jr (2012): High-dose curcuminoids are efficacious in the reduction in symptoms and signs of oral lichen planus. $\mathrm{J}$ Am Acad Dermatol. May;66(5):752-60. doi: 10.1016/j. jaad.2011.04.022. Epub 2011 Sep 9. PubMed PMID: 21907450 .

29. Amirchaghmaghi M, Pakfetrat A, Delavarian Z, Ghalavani H, Ghazi A (2016): Evaluation of the Efficacy of Curcumin in the Treatment of Oral Lichen Planus: A Randomized Controlled Trial. J Clin Diagn Res;10(5):ZC134-7. doi: 10.7860/JCDR/2016/16338.7870. Epub 2016 May 1. PubMed PMID: 27437348; PubMedCentral PMCID: PMC4948524.

30. Bramara Kumari K, Satya Bhushan NVV, Sunil T, Siva Kalyan U, Kho Chai C, Srinivas Saketh G, Bharat Prakash M (2017): Efficacy of turmeric in the management of oral lichen planus. International Journal of Current Research; 9(03): 48119-48124.

31. Singh V, Singh G, Pal M, Tandon K (2017): Treatment of Lichen Planus: A Comparative Study. J Tradit Med Clin Natur 6: 246.

32. Keshari D, Patil K, Mahima VG (2015): Efficacy of topical curcumin in the management of oral lichen planus: A randomized controlled-trial. Journal of Advanced Clinical \& Research Insights, 2, 197-203.

33. Kia SJ, Shirazian S, Mansourian A, Khodadadi Fard L, Ashnagar S (2015): Comparative Efficacy of Topical Curcumin and Triamcinolone for Oral Lichen Planus: A Randomized, Controlled Clinical Trial. J Dent (Tehran); 12(11):789-96. PubMed PMID: 27507989; PubMed Central PMCID: PMC4977402.

34. Thomas AE, Varma B, Kurup S, Jose R, Chandy ML, Kumar SP, Aravind MS, Ramadas AA (2017): Evaluation of Efficacy of $1 \%$ Curcuminoids as Local Application in Management of Oral Lichen Planus - Interventional Study. J Clin Diagn Res; 11(4):ZC89-ZC93. doi: 10.7860/ JCDR/2017/20898.9715. PubMed PMID: 28571271; PubMed Central PMCID: PMC5449927.

35. Nosratzehi T, Arbabi-Kalati F, Hamishehkar H, Bagheri S (2018): Comparison of the Effects of Curcumin Mucoadhesive Paste and Local Corticosteroid on the Treatment of Erosive Oral Lichen Planus Lesions. J Natl Med Assoc.; 110(1):92-97. doi: 10.1016/j. jnma.2017.01.011. PubMed PMID: 29510851.

36. Chainani-Wu N, Silverman S Jr, Reingold A, Bostrom A, Lozada-Nur F, Weintraub J (2008): Validation of instruments to measure the symptoms and signs of oral lichen planus. Oral Surg Oral Med Oral Pathol Oral Radiol Endod; 105(1):51-8. PubMed PMID: 18155609.

37. Scott J, Huskisson EC (1976): Graphic representation of pain. Pain; 2(2):175-84.

38. Thongprasom K, Luangjarmekorn L, Sererat T, Taweesap W (1992): Relative efficacy of fluocinolone acetonide compared with triamcinolone acetonide in treatment of oral lichen planus. J Oral Pathol Med; 21(10):456-8.

39. Williamson A, Hoggart B (2005): Pain: A review of three commonly used pain rating scales. J Clin Nurs; 14:798804.

40. Schubert MM, Williams BE, Lloid ME, Donaldson G, Chapko MK (1992): Clinical assessment scale for the rating of oral mucosal changes associated with bone marrow transplantation: Development of an oral mucositis index. Cancer; 69:2469-77.

41. Amirchaghmaghi, M, Delavarian, Z, Iranshahi, M, \& Shakeri, M (2015): A randomized placebo-controlled double blind clinical trial of quercetin for treatment of oral lichen planus. J Dent Res Dent Clin Dent Prosp; 9(1): 23-28.

42. Piboonniyom S O, Treister N, Pitiphat W and Woo S B (2005): Scoring system for monitoring oral lichenoid lesions: a preliminary study. Oral Surg Oral Med Oral Pathol Oral Radiol Endod, 99, 696-703.

43. Thongprasom K, Carrozzo M, Furness S and Lodi G (2011): Interventions for treating oral lichen planus. Cochrane Database Syst Rev; 7: CD001168.

44. Cheng S, Kirtschig G, Cooper S, Thornhill M, LeonardiBee J, Murphy R (2012): Interventions for erosive lichen planus affecting mucosal sites. Cochrane Database Syst Rev; 2: CD008092. 
45. Lodi G, Carrozzo M, Furness S, Thongprasom K (2012): Interventions for treating oral lichen planus: a systematic Review. Br J Dermatol 166: 938-947.

46. Gorouhi F, Solhpour A, Beitollahi JM, Afshar S, Davari P, Hashemi P, Nassiri Kashani M, Firooz A. (2007): Randomized trial of pimecrolimus cream versus triamcinolone acetonide paste in the treatment of oral lichen planus. J Am Acad Dermatol; 57: 806-13

47. Arduino PG, Carbone M, Della Ferrera F, Elia A, Conrotto D, Gambino A, Comba A, Calogiuri PL, Broccoletti R (2013): Pimecrolimus vs. tacrolimus for the topical treatment of unresponsive oral erosive lichen planus: a 8 week randomized double-blind controlled study. J Eur Acad Dermatol Venereol; 28(4):475-82. doi:10.1111/ jdv.12128.
48. Wu Y, Zhou G, Zeng H, Xiong CR, Lin M, Zhou HM (2010): A randomized double-blind positive control trial of topical thalidomide in erosive oral lichen planus. Oral Surg Oral Med Oral Pathol Oral Radiol Endod; 110: 188-95.

49. Fu J, Zhu X, Dan H, Zhou Y, Liu C, Wang F, Li Y, Liu N, Chen Q, Xu Y, 'Zeng X, Jiang L (2012): Amlexanox is as effective as dexamethasone in topical treatment of erosive oral lichen planus: a short-term pilot study. Oral Surg Oral Med Oral Pathol Oral Radiol; 113: 638-43.

50. Reddy RL, Reddy RS, Ramesh T, Singh TR, Swapna LA, Laxmi NV (2012): Randomized trial of aloe vera gel vs triamcinolone acetonide ointment in the treatment of oral lichen planus. Quintessence Int; 43:793-800. 